\title{
A Note on the Appearance of Self-Dual Yang-Mills Fields in Integrable Hierarchies
}

\author{
L. FEHÉR and A. GÁBOR \\ Department of Theoretical Physics, University of Szeged, \\ Tisza Lajos krt 84-86, H-6720 Szeged, Hungary \\ E-mail: lfeher@sol.cc.u-szeged.hu \\ Received February 11, 2000; Accepted June 15, 2000
}

\begin{abstract}
A family of mappings from the solution spaces of certain generalized Drinfeld-Sokolov hierarchies to the self-dual Yang-Mills system on $\mathbf{R}^{2,2}$ is described. This provides an extension of the well-known relationship between self-dual connections and integrable hierarchies of AKNS and Drinfeld-Sokolov type.
\end{abstract}

\section{Introduction}

The four-dimensional self-dual Yang-Mills equations have several important applications in physics and mathematics [1]. For example, many lower dimensional integrable systems can be obtained as reductions of the self-dual Yang-Mills system, which represents a method to classify integrable systems and to apply twistor techniques to study them. Research in this direction was initiated by Ward [2], the subsequent investigations are reviewed in [3, 4]. Of particular interest for us is the relationship between the self-dual Yang-Mills system and integrable hierarchies of $(1+1)$-dimensional soliton equations. It has been shown by Mason and collaborators that the nonlinear Schrödinger and the Korteweg - de Vries equations [5] as well as the $n-\mathrm{KdV}$ hierarchies [6] are reductions of the self-dual Yang-Mills system. The generalized AKNS hierarchies [7] and the modified KdV systems of Drinfeld-Sokolov [8] have been connected to the self-dual Yang-Mills system in $[9,10,11]$, and super-extensions are discussed in [12].

It is expected that the set of hierarchies related to the self-dual Yang-Mills system is larger than those mentioned above, and should include many of the generalized DrinfeldSokolov hierarchies introduced in [13]. To show that this is indeed the case, in this letter we exhibit a family of mappings from the solutions of a large class of integrable hierarchies to those of the self-dual Yang-Mills equations. We shall be concerned with self-dual Yang-Mills fields on $\mathbf{R}^{4}$ equipped with a pseudo-Euclidean metric of signature $(2,2)$. The 
coordinates on this space are denoted by $y^{\mu}(\mu=1,2,3,4)$ and the flat metric $\eta_{\mu \nu}$ is given as

$$
\eta_{\mu \nu}=\left[\begin{array}{cccc}
0 & 0 & 1 & 0 \\
0 & 0 & 0 & -1 \\
1 & 0 & 0 & 0 \\
0 & -1 & 0 & 0
\end{array}\right]
$$

A Yang-Mills field is represented by a connection

$$
D_{\mu}=\frac{\partial}{\partial y^{\mu}}+A_{\mu}
$$

where $A_{\mu}(y)$ varies in some Lie algebra $\mathcal{G}$. By using $F_{\mu \nu}=\left[D_{\mu}, D_{\nu}\right]$ and the usual LeviCivita tensor for which $\epsilon_{1234}=1$, the self-duality equations are defined by

$$
F_{\mu \nu}=\frac{1}{2} \epsilon_{\mu \nu \alpha \beta} F^{\alpha \beta},
$$

where the indices are raised by the inverse metric $\eta^{\mu \nu}$. Explicitly, they read as

$$
F_{12}=0, \quad F_{34}=0, \quad F_{13}=F_{24} .
$$

Equivalently, self-duality can be characterized by the requirement that the operators

$$
\begin{aligned}
& P:=D_{1}-\lambda D_{4}=\frac{\partial}{\partial y^{1}}+A_{1}(y)-\lambda\left(\frac{\partial}{\partial y^{4}}+A_{4}(y)\right) \\
& P^{\prime}:=D_{2}-\lambda D_{3}=\frac{\partial}{\partial y^{2}}+A_{2}(y)-\lambda\left(\frac{\partial}{\partial y^{3}}+A_{3}(y)\right)
\end{aligned}
$$

satisfy

$$
\left[P, P^{\prime}\right]=0
$$

at any value of the spectral parameter $\lambda$. We shall see below that commuting operators of the form $P, P^{\prime}$ above naturally arise from a class of integrable hierarchies which contains, besides the AKNS and Drinfeld-Sokolov hierarchies associated with the untwisted affine Lie algebras, many other special cases of the systems introduced in [13].

\section{Self-dual Yang-Mills from integrable hierarchies}

We next review the basics of the construction of a general class of integrable hierarchies. For a more detailed description, see e.g. [13, 14]. Then we shall specialize the input data of the construction in such a way that the solutions of the hierarchy give rise to solutions of the self-dual Yang-Mills equation.

The input data of the subsequent construction is a triplet $(\mathcal{A}, D, \Lambda)$, where $\mathcal{A}$ is an infinite dimensional Lie algebra, $D$ is a derivation that defines a $\mathbf{Z}$-gradation of $\mathcal{A}$, and $\Lambda$ is a semisimple element of $\mathcal{A}$ which is homogeneous of degree $k>0$ in the gradation $D$. In more detail, the gradation is given by the eigenspaces $\mathcal{A}^{n}$ of the derivation $D: \mathcal{A} \rightarrow \mathcal{A}$,

$$
\mathcal{A}=\oplus_{n \in \mathbf{Z}} \mathcal{A}^{n} \quad \text { with } \quad \mathcal{A}^{n}=\{X \in \mathcal{A} \mid D(X)=n X\} .
$$


One can restrict the sum to the spectrum of $D$ consisting of the grades for which $\mathcal{A}^{n} \neq\{0\}$, and we suppose that $\operatorname{dim}\left(\mathcal{A}^{n}\right)<\infty$. As a consequence of $D[X, Y]=[D X, Y]+[X, D Y]$, one has $\left[\mathcal{A}^{m}, \mathcal{A}^{n}\right] \subset \mathcal{A}^{m+n}$. The semisimplicity of $\Lambda \in \mathcal{A}$ means that it yields a linear direct sum decomposition as

$$
\mathcal{A}=\operatorname{Ker}(\operatorname{ad} \Lambda)+\operatorname{Im}(\operatorname{ad} \Lambda), \quad \operatorname{Ker}(\operatorname{ad} \Lambda) \cap \operatorname{Im}(\operatorname{ad} \Lambda)=\{0\},
$$

where

$$
\operatorname{Ker}(\operatorname{ad} \Lambda)=\{X \in \mathcal{A} \mid[\Lambda, X]=0\}, \quad \operatorname{Im}(\operatorname{ad} \Lambda)=\{X \in \mathcal{A} \mid X=[\Lambda, Y] \text { for } Y \in \mathcal{A}\} .
$$

The assumption that $D(\Lambda)=k \Lambda$ ensures that $\operatorname{Ker}(\operatorname{ad} \Lambda)$ is a graded Lie subalgebra of $\mathcal{A}$ and $\operatorname{Im}(\operatorname{ad} \Lambda)$ is a graded vector space.

Given the above data, one can define an infinite set of commuting evolutional vector fields on the 'phase space'

$$
\mathcal{M}_{\Lambda, D}:=\left\{L=\partial_{x}+j(x)+\Lambda \mid j(x) \in \mathcal{A}^{<k}\right\}
$$

Here $\mathcal{A}^{<k}=\oplus_{n<k} \mathcal{A}^{n}$ and analogous notations will be used without further explanation. The dynamical variables are the infinitely many independent component fields of $j(x)$, which are smooth (real or complex valued depending on whether $\mathcal{A}$ is a real or a complex Lie algebra) functions of the one-dimensional 'space variable' $x$. The evolutional vector fields are associated with the non-negatively graded part $\mathcal{H}_{\Lambda}^{+}$of the centre of $\operatorname{Ker}(\operatorname{ad} \Lambda)$ :

$$
\mathcal{H}_{\Lambda}^{+}=\{c \in \operatorname{Ker}(\operatorname{ad} \Lambda) \mid[c, X]=0 \quad \forall X \in \operatorname{Ker}(\operatorname{ad} \Lambda)\} \cap \mathcal{A}^{\geq 0} .
$$

Concretely, for any $c \in \mathcal{H}_{\Lambda}^{+}$one obtains a vector field $V_{c}$ on $\mathcal{M}_{\Lambda, D}$ as follows. First, for any $L \in \mathcal{M}_{\Lambda, D}$ one considers the Drinfeld-Sokolov dressing transformation:

$$
L=\left(\partial_{x}+j(x)+\Lambda\right) \mapsto e^{\operatorname{ad} F}(L)=\left(\partial_{x}+h(x)+\Lambda\right),
$$

where $F(x)$ and $h(x)$ are supposed to be formal series

$$
F(x) \in \operatorname{Im}(\operatorname{ad} \Lambda)^{<0}, \quad h(x) \in(\operatorname{Ker}(\operatorname{ad} \Lambda))^{<k} .
$$

It is well-known $[8,13]$ that there exists a unique solution $F(x)=F(j(x)), h(x)=h(j(x))$ of (2.6) for any $L$, and the components of $F$ and $h$ are finite polynomials in the components of $j(x)$ and their $x$-derivatives. We here use

$$
e^{\operatorname{ad} F}(L)=\sum_{n=0}^{\infty} \frac{1}{n !}(\operatorname{ad} F)^{n}(L) \quad \text { with } \quad(\operatorname{ad} F)(L)=-\partial_{x} F+[F, j+\Lambda],
$$

or, equivalently, $e^{\text {adF }}(L)$ may be thought of as $e^{F} L e^{-F}$. The vector field $V_{c}$ is defined by its action on the 'coordinate' $j$ on $\mathcal{M}_{\Lambda, D}$ as follows:

$$
V_{c} j(x):=-\partial_{x} B(c, j(x))^{\geq 0}+\left[B(c, j(x))^{\geq 0}, j(x)+\Lambda\right],
$$

where

$$
B(c, j):=e^{-\operatorname{ad} F(j)}(c) \quad \forall c \in \mathcal{H}_{\Lambda}^{+},
$$


and $B(c, j)=B(c, j)^{\geq 0}+B(c, j)^{<0}$ according to the gradation $D$. Since $\left[B(c, j)^{\geq 0}, L\right]=$ $-\left[B(c, j)^{<0}, L\right], V_{c}$ is a well-defined vector field on $\mathcal{M}_{\Lambda, D}$, that is, $V_{c}(j(x)) \in \mathcal{A}^{<k}$. Since $\mathcal{H}_{\Lambda}^{+}$is an Abelian Lie algebra, the vector fields $V_{c}$ commute among themselves,

$$
\left[V_{c}, V_{c^{\prime}}\right](j(x))=0 \quad \forall c, c^{\prime} \in \mathcal{H}_{\Lambda}^{+}
$$

If we introduce a time variable $t^{c}$ for every basis element $c \in \mathcal{H}_{\Lambda}^{+}$, and denote the collection of these variables by $\mathbf{t}$, then we can write the evolution equations defined by the $V_{c}$,

$$
\frac{\partial}{\partial t^{c}} j(x, \mathbf{t})=-\partial_{x} B(c, j(x, \mathbf{t}))^{\geq 0}+\left[B(c, j(x, \mathbf{t}))^{\geq 0}, j(x, \mathbf{t})+\Lambda\right],
$$

in the zero curvature form

$$
\left[\mathcal{L}_{c}, L\right]=0 \quad \text { with } \quad L=\partial_{x}+j(x, \mathbf{t})+\Lambda, \quad \mathcal{L}_{c}=\frac{\partial}{\partial t^{c}}-B(c, j(x, \mathbf{t}))^{\geq 0} .
$$

As a result of the compatibility (2.11) of these evolution equations, one then also has

$$
\left[\mathcal{L}_{c}, \mathcal{L}_{c^{\prime}}\right]=0 \quad \forall c, c^{\prime} \in \mathcal{H}_{\Lambda}^{+} .
$$

After this preparation, we now show that under certain choices of the data the above zero curvature equations imply the self-duality equations described in the introduction. Since we wish to obtain commuting operators of the form in (1.5), we take $\mathcal{A}$ to be a loop algebra,

$$
\mathcal{A}=\mathcal{G} \otimes \mathbf{C}\left(\left(\lambda^{-1}\right)\right)=\left\{X=\sum_{i \leq N_{X}} X_{i} \lambda^{i} \mid X_{i} \in \mathcal{G}, N_{X} \in \mathbf{Z}\right\}
$$

where $\mathcal{G}$ is a finite dimensional Lie algebra. We assume that the gradation $D$ of $\mathcal{A}$ is given by

$$
D=M \lambda \frac{d}{d \lambda}+\operatorname{ad} H
$$

where $H$ is an ad-diagonalizable element of $\mathcal{G}$ with integer eigenvalues whose absolute values are strictly less than $M \in \mathbf{N}$. Thus we have a corresponding gradation of $\mathcal{G}$,

$$
\mathcal{G}=\oplus_{n} \mathcal{G}^{n} \quad \text { where } \quad|n|<M .
$$

As before, $\Lambda \in \mathcal{A}$ is a semisimple element of $D$-grade $k>0$. Under these assumptions, we can introduce a basis $c^{a, l}$ of $\mathcal{H}_{\Lambda}^{+}$given by

$$
c^{a, l}:=\lambda^{l} c^{a} \quad \text { with } \quad c^{a} \in \mathcal{H}_{\Lambda}^{+} \cap \mathcal{A}^{<M}, \quad \text { and } \quad l=0,1,2, \ldots,
$$

where $c^{a}=c^{a, 0}$ is a basis of $\mathcal{H}_{\Lambda}^{+} \cap \mathcal{A}^{<M}$. We shall denote the time variables $t^{c}$ and the Lax operators $\mathcal{L}_{c}$ corresponding to these base elements respectively as $t^{a, l}$ and $\mathcal{L}_{a, l}$. The general construction now implies that

$$
B\left(c^{a, l}, j\right)=\lambda^{l} B\left(c^{a}, j\right),
$$


and it follows from our assumption on the gradation of $\mathcal{G}$ in $(2.17)$ that $B\left(c^{a}, j\right)$ expands as

$$
B\left(c^{a}, j\right)=\sum_{i \leq 1} B_{i}\left(c^{a}, j\right) \lambda^{i}, \quad B_{i}\left(c^{a}, j\right) \in \mathcal{G}
$$

By using these expansions, the Lax operators defining the hierarchy can be written as

$$
\mathcal{L}_{a, l}=\frac{\partial}{\partial t^{a, l}}-B_{-l}\left(c^{a}, j(x, \mathbf{t})\right)^{\geq 0}-\sum_{i=1-l}^{1} \lambda^{i+l} B_{i}\left(c^{a}, j(x, \mathbf{t})\right) \quad \forall l \geq 0 .
$$

In particular, these operators contain only non-negative powers of $\lambda$, due to our assumption on the eigenvalues of $\operatorname{ad} H$ in (2.17). We then notice that the operators $P_{a, l}:=\mathcal{L}_{a, l+1}-\lambda \mathcal{L}_{a, l}$ have the same form as the Lax operators of the self-dual Yang-Mills equations in (1.5). Indeed, we obtain

$$
P_{a, l}=\frac{\partial}{\partial t^{a, l+1}}-B_{-l-1}\left(c^{a}, j(x, \mathbf{t})\right)^{\geq 0}-\lambda\left(\frac{\partial}{\partial t^{a, l}}+B_{-l}\left(c^{a}, j(x, \mathbf{t})\right)^{<0}\right) \quad \forall l \geq 0
$$

where $B_{i}\left(c^{a}, j(x, \mathbf{t})\right)^{\geq 0}$ (resp. $\left.B_{i}\left(c^{a}, j(x, \mathbf{t})\right)^{<0}\right)$ is the part of $B_{i}\left(c^{a}, j(x, \mathbf{t})\right) \in \mathcal{G}$ defined by the decomposition $\mathcal{G}=\mathcal{G}^{\geq 0}+\mathcal{G}^{<0}$ associated with (2.17). Moreover, it is convenient to introduce 'cyclic coordinates' $t^{a,-1}$, on which nothing depends, since by using them we may write

$$
P_{a,-1}:=\mathcal{L}_{a, 0}-\lambda \frac{\partial}{\partial t^{a,-1}}=\frac{\partial}{\partial t^{a, 0}}-B_{0}\left(c^{a}, j(x, \mathbf{t})\right)^{\geq 0}-\lambda\left(\frac{\partial}{\partial t^{a,-1}}+B_{1}\left(c^{a}, j(x, \mathbf{t})\right)\right),
$$

which formally follows the same pattern as the other $P_{a, l}$. Note that $B_{1}\left(c^{a}, j(x, \mathbf{t})\right) \in \mathcal{G}^{<0}$.

Let us now choose pairs of indices $a, l$ and $a^{\prime}, l^{\prime}$ in such a way that the set

$$
y^{1}:=t^{a, l+1}, \quad y^{4}:=t^{a, l}, \quad y^{2}:=t^{a^{\prime}, l^{\prime}+1}, \quad y^{3}:=t^{a^{\prime}, l^{\prime}}
$$

consists of 4 different coordinates. The main point of this paper is the observation that any solution $j(x, \mathbf{t})$ of the above constructed integrable hierarchy gives rise to self-dual Yang-Mills fields by the mapping

$$
P_{a, l} \mapsto P, \quad P_{a^{\prime}, l^{\prime}} \mapsto P^{\prime} .
$$

To obtain self-dual Yang-Mills fields by this correspondence, it is enough that the evolution equations of the hierarchy be satisfied for the finite subset of the variables given by $x$ and the $t$ 's that occur in (2.24). On the other hand, if $j(x, \mathbf{t})$ satisfies the hierarchy in all (or many) time variables, then it gives rise to (infinitely) many self-dual Yang-Mills fields, too, since the identification of the $y^{\mu}$ 's in terms of the $t$ 's is then possible in several different ways. Note that if $P_{a,-1}$ is taken as one of the Lax operators, then the corresponding YangMills fields are invariant under the translations of the cyclic coordinate $t^{a,-1}$, and thus the component $B_{1}\left(c^{a}, j(x, \mathbf{t})\right)$ associated with this coordinate in $(2.23)$ can be regarded as 
a 'Higgs field' in the adjoint representation of $\mathcal{G}$. In these cases the self-duality equation (1.6) becomes an analogue of the Bogomolny equation (see e.g. [4]).

The self-dual Yang-Mills fields that appear in the above are special since they vary in the subalgebras $\mathcal{G}^{\geq 0}$ and $\mathcal{G}^{<0}$ of $\mathcal{G}$. If $D$ is a multiple of the homogeneous gradation, $D=M \lambda \frac{d}{d \lambda}$, then $\mathcal{G}^{\geq 0}=\mathcal{G}$ and $\mathcal{G}^{<0}=\{0\}$, and in this case another special feature is that the Yang-Mills potentials that multiply $\lambda$ in $P_{a, l}$ vanish. The Yang-Mills potentials that arise from (2.25) are differential polynomials in the infinitely many components of the field $j(x, \mathbf{t}) \in \mathcal{A}^{<k}$ contained in the basic Lax operator $L$ of the hierarchy. After reducing the degrees of freedom to finitely many fields, in certain cases $j$ itself can be regarded as a $\mathcal{G}$-valued Yang-Mills potential. These reductions are discussed briefly in the next section.

\section{Reductions, gauge transformations and examples}

It is not difficult to check that the vector fields $V_{c}(2.9)$ are always tangential to the submanifold $\Theta_{\Lambda, D} \subset \mathcal{M}_{\Lambda, D}$ defined by

$$
\Theta_{\Lambda, D}:=\left\{L=\partial_{x}+j(x)+\Lambda \mid j(x) \in \mathcal{A}^{<k} \cap \mathcal{A}^{\geq 0}\right\}
$$

The restriction of the evolution equations (2.12) to this phase space, where $j$ has only finitely many components, is called a modified KdV type hierarchy [13]. Among the modified KdV type hierarchies that can be mapped to the self-dual Yang-Mills system, let us now concentrate on those for which the condition

$$
D(\Lambda)=k \Lambda \quad 0<k \leq M
$$

holds besides eqs. (2.15), (2.16), (2.17). Then, introducing also another cyclic coordinate $z$, we can represent the basic Lax operator $L$ as

$$
L=\frac{\partial}{\partial x}+\left(j_{0}(x, \mathbf{t})+\Lambda_{0}\right)-\lambda\left(\frac{\partial}{\partial z}-j_{1}(x, \mathbf{t})-\Lambda_{1}\right)
$$

since we have the decompositions $\Lambda=\left(\Lambda_{0}+\Lambda_{1} \lambda\right)$ and $j=\left(j_{0}+j_{1} \lambda\right)$ with some $\Lambda_{i}, j_{i} \in \mathcal{G}$. Notice that this operator now has the same form as those in (1.5). Therefore it follows from (2.13) that we obtain self-dual Yang-Mills fields from the solutions of the hierarchy on $\Theta_{\Lambda, D}$ not only by the correspondence in (2.25), but also via the mapping

$$
P_{a, l} \mapsto P, \quad L \mapsto P^{\prime},
$$

whereby $\left(j_{0}+\Lambda_{0}\right)$ and $-\left(j_{1}+\Lambda_{1}\right)$ become components of the Yang-Mills potential. More precisely, $\left(j_{1}+\Lambda_{1}\right)$ can be regarded as a Higgs field since nothing depends on the associated space-time coordinate $y^{3}=z$. Examples of this kind are the generalized AKNS systems for which $k=M=1$ with $D=\lambda \frac{d}{d \lambda}, \Lambda=\lambda \Lambda_{1}$. The mapping of the solutions of the hierarchy to self-dual connections has in this case been described previously in [9]. Another set of examples is furnished by the generalized modified KdV systems of Drinfeld-Sokolov [8] associated with the untwisted affine Lie algebras. For these systems $D$ is the so called principal gradation and $\Lambda$ has grade $k=1<M$, where $M$ is the dual Coxeter number of a simple Lie algebra $\mathcal{G}$. 
A different class of reductions of the systems on $\mathcal{M}_{\Lambda, D}$ are the $\mathrm{KdV}$ type hierarchies $[8,13]$. Their construction uses, in addition to $\mathcal{A}$ in $(2.15), D$ in $(2.16)$ and $\Lambda \in \mathcal{A}$ of $D$ grade $k>0$, an auxiliary integral gradation of $\mathcal{A}$. Let this be provided by the eigenvalues of the derivation

$$
d=m \lambda \frac{d}{d \lambda}+\operatorname{ad} h \quad(m \in \mathbf{N}, h \in \mathcal{G}) .
$$

The two gradations are compatible, $[D, d]=0$, and must satisfy

$$
\mathcal{A}^{D>0} \subset \mathcal{A}^{d \geq 0}, \quad \mathcal{A}^{D=0} \subset \mathcal{A}^{d=0}, \quad \mathcal{A}^{D<0} \subset \mathcal{A}^{d \leq 0} .
$$

Furthermore, one assumes that

$$
\Gamma:=\mathcal{A}^{D<0} \cap \mathcal{A}^{d=0}
$$

is a non-trivial subalgebra of $\mathcal{A}$ for which

$$
\Gamma \cap \operatorname{Ker}(\operatorname{ad} \Lambda)=\{0\} .
$$

For our purpose, we also require that $|\operatorname{ad} h|<m$, which means that the ad $h$-grades in $\mathcal{G}$ are strictly smaller than $m$ (analogously to the property of $D$ in (2.17)). As a consequence, we have $\Gamma \subset \mathcal{G}$, where $\mathcal{G}$ is naturally embedded in $\mathcal{A}$ as the set of $\lambda$-independent elements.

To associate a $\mathrm{KdV}$ type hierarchy with the data $(\mathcal{A}, \Lambda, D, d)$, one first restricts the hierarchy on $\mathcal{M}_{\Lambda, D}$ to the submanifold

$$
\mathcal{M}_{\Lambda, D, d}:=\left\{L=\partial_{x}+j(x)+\Lambda \mid j(x) \in \mathcal{A}^{D<k} \cap \mathcal{A}^{d \geq 0}\right\} \subset \mathcal{M}_{\Lambda, D} .
$$

The above conditions guarantee that the restriction is consistent. That is the vector fields $V_{c}(2.9)$, still defined by means of the $D$-gradation, are in our case tangential to $\mathcal{M}_{\Lambda, D, d}$. The next step is to consider the transformations

$$
L=\left(\partial_{x}+j(x)+\Lambda\right) \mapsto L^{\gamma}=e^{\gamma(x)} L e^{-\gamma(x)}=\left(\partial_{x}+j^{\gamma}(x)+\Lambda\right) \quad \forall \gamma(x) \in \Gamma .
$$

Explicitly,

$$
j^{\gamma}(x)=e^{\gamma(x)}(j(x)+\Lambda) e^{-\gamma(x)}-\Lambda-\left(\partial_{x} e^{\gamma(x)}\right) e^{-\gamma(x)} .
$$

These transformations define an action of the group $\mathcal{N}:=\left\{e^{\gamma} \mid \gamma(x) \in \Gamma\right\}$ on $\mathcal{M}_{\Lambda, D, d}$, and one sees from (3.8) that this is a free action, i.e., only the unit element of $\mathcal{N}$ has fixed points. Then $\mathcal{N}$ is regarded as a group of gauge transformations for the hierarchy on $\mathcal{M}_{\Lambda, D, d}$, which is possible because the vector field $V_{c}(2.9)$ has a well-defined projection on the factor space $\mathcal{M}_{\Lambda, D, d} / \mathcal{N}$. This follows from the relations

$$
B\left(c, j^{\gamma}\right)=e^{\gamma} B(c, j) e^{-\gamma} \quad \text { and } \quad\left(e^{\gamma} B(c, j)^{D \geq 0} e^{-\gamma}-B\left(c, j^{\gamma}\right)^{D \geq 0}\right) \in \Gamma,
$$

which are consequences of the definition of $B(c, j)(2.10)$ and eqs. (3.6), (3.8). By definition, the KdV type hierarchy is the hierarchy on $\mathcal{M}_{\Lambda, D, d}$ modulo the gauge group $\mathcal{N}$.

As this is known to hold in special cases $[5,6]$, it is natural to ask whether ' $\mathrm{KdV}$ gauge equivalence' always implies gauge equivalence of the corresponding self-dual Yang-Mills fields. To answer this question, consider a time dependent gauge transformation of a 
solution of the hierarchy on $\mathcal{M}_{\Lambda, D, d}$. The solution represented by $L(\mathbf{t})=\left(\partial_{x}+j(x, \mathbf{t})+\Lambda\right)$ is sent to $L^{\gamma}(\mathbf{t})=\left(\partial_{x}+j^{\gamma}(x, \mathbf{t})+\Lambda\right)$, where we apply (3.10) with a function $\gamma(x, \mathbf{t}) \in \Gamma$. The definition of the hierarchy implies that $j^{\gamma}(x, \mathbf{t})$ will be another solution if and only if $\gamma(x, \mathbf{t})$ solves the following evolution equations:

$$
\frac{\partial e^{\gamma(x, \mathbf{t})}}{\partial t^{c}} e^{-\gamma(x, \mathbf{t})}=B\left(c, j^{\gamma}(x, \mathbf{t})\right)^{D \geq 0}-e^{\gamma(x, \mathbf{t})} B(c, j(x, \mathbf{t}))^{D \geq 0} e^{-\gamma(x, \mathbf{t})} .
$$

By using (3.12) and (2.11), one can check that (3.13) yields well-defined, compatible evolution equations on $\gamma(x, \mathbf{t})$. In conclusion, the solutions of the hierarchy on $\mathcal{M}_{\Lambda, D, d}$ which are gauge equivalent to $L(\mathbf{t})$ have the form $L^{\gamma}(\mathbf{t})$ where $\gamma(x, \mathbf{t})$ solves (3.13) for some initial value $\gamma(x)$ at $\mathbf{t}=0$. One easily sees from this that the Lax operators $\mathcal{L}_{c}$ defined in (2.13) behave in the following manner with respect to the gauge transformations of the solutions:

$$
\begin{aligned}
\mathcal{L}_{c}= & \left(\frac{\partial}{\partial t^{c}}-B(c, j(x, \mathbf{t}))^{D \geq 0}\right) \mapsto \\
& \mathcal{L}_{c}^{\gamma}:=\left(\frac{\partial}{\partial t^{c}}-B\left(c, j^{\gamma}(x, \mathbf{t})\right)^{D \geq 0}\right)=e^{\gamma(x, \mathbf{t})} \mathcal{L}_{c} e^{-\gamma(x, \mathbf{t})} .
\end{aligned}
$$

This formula permits us to show that the self-dual Yang-Mills fields corresponding, either $^{1}$ by (2.25) or by (3.4), to gauge equivalent solutions of the hierarchy on $\mathcal{M}_{\Lambda, D, d}$ are always gauge equivalent in the usual Yang-Mills sense. The equivalence of two $\mathcal{G}$-valued Yang-Mills connections $D_{\mu}$ and $\tilde{D}_{\mu}$ requires the existence of a function $g(y)$ in the group generated by $\mathcal{G}$ for which $\tilde{D}_{\mu}=g(y) D_{\mu} g^{-1}(y)$. In the self-dual case, this amounts to $\tilde{P}(y)=g(y) P(y) g^{-1}(y)$ and to $\tilde{P}^{\prime}(y)=g(y) P^{\prime}(y) g^{-1}(y)$ for the operators in (1.5). Now it is obvious from (3.14) and (2.22), (2.23) that if $D_{\mu}$ belongs to $j(x, \mathbf{t})$ and $\tilde{D}_{\mu}$ to $j^{\gamma}(x, \mathbf{t})$ then they are gauge related by $g(y)=e^{\gamma(x, \mathbf{t})}$, where the arguments of $\gamma(x, \mathbf{t})$ different from the $y^{\mu}$ are fixed arbitrarily. As mentioned above, $\Gamma \subset \mathcal{G}$, and thus the proof is complete.

The conditions used in the above are satisfied, for example, for the generalized $\mathrm{KdV}$ hierarchies associated with the regular conjugacy classes of the Weyl groups of the simple Lie algebras [15]. These are special cases of the systems introduced in [13], and contain the untwisted Drinfeld-Sokolov hierarchies, which belong to the Coxeter conjugacy class. Most of them can be described alternatively as constrained (matrix) KP hierarchies. As one of the simplest examples, let us mention the much studied constrained KP hierarchies that have scalar Lax operators of the form

$$
L_{\mathrm{cKP}}=\partial^{n}+u_{1} \partial^{n-1}+\cdots+u_{n}+v_{+}(\partial+w)^{-1} v_{-}
$$

for some $n \geq 1$. These systems are associated with $g l_{n+1}$ and the elements of the corresponding conjugacy class of the Weyl group $S_{n+1}$ decompose as the product of a $n$-cycle and a 1-cycle. For detailed description, see e.g. [16] and references therein. It would be interesting to further explore the nature of the relationship between these hierarchies and the self-dual Yang-Mills equations. For instance, following the spirit of $[4,5,6]$, it should be possible to view at least some of them as reductions of a self-dual Yang-Mills hierarchy, and to apply twistor techniques to describe their $\tau$-functions.

\footnotetext{
${ }^{1}$ Due to our assumptions on the eigenvalues of $\operatorname{ad} H$ and $\operatorname{ad} h$, the mapping (3.4) can be used if $k \leq M$ is valid for the $\mathrm{KdV}$ type system.
} 
Finally, we wish to remark that there exist certain constrained modified KP (also called non-standard $n$-KdV) hierarchies that fit into an extended version of the Drinfeld-Sokolov approach for which our above assumptions do not hold [17]. These systems, and also the Drinfeld-Sokolov hierarchies based on the twisted affine Lie algebras, cannot be mapped to the self-dual Yang-Mills system by the method presented here. In fact, in these cases the analogues of $P_{a, l}=\mathcal{L}_{a, l+1}-\lambda \mathcal{L}_{a, l}$ in (2.22) would contain both positive and negative, or higher than first powers of the spectral parameter, which do not appear in (1.5).

\section{Acknowledgements}

This work has been supported in part by the Hungarian Ministry of Education under FKFP 0596/1999 and by the National Science Fund (OTKA) under T025120.

\section{References}

[1] Ward R.S. and Wells R.O., Twistor Geometry and Field Theory, Cambridge University Press, Cambridge, 1990.

[2] Ward R.S., Integrable and Solvable Systems and Relations Among Them, Phil. Trans. Roy. Soc. Lond. A, 1985, V.315, 451-457.

[3] Ablowitz M.J. and Clarkson P.A., Solitons, Nonlinear Evolution Equations and Inverse Scattering, Cambridge University Press, Cambridge, 1991.

[4] Mason L.J. and Woodhouse N.M.J., Integrability, Self-Duality, and Twistor Theory, Clarendon Press, Oxford, 1996.

[5] Mason L.J. and Sparling G.A.J., Nonlinear Schrödinger and Korteweg de Vries are Reductions of Self-Dual Yang-Mills, Phys. Lett. A, 1989, V.137, 29-33; Twistor Correspondences for the Soliton Hierarchies, J. Geom. Phys., 1992, V.8, 243-271.

[6] Mason L.J. and Singer M.A., The Twistor Theory of Equations of KdV Type. I, Commun. Math. Phys., 1994, V.166, 191-218.

[7] Wilson G.W., The Modified Lax and Two-Dimensional Toda Lattice Equations Associated with Simple Lie Algebras, Ergod. Th. and Dynam. Sys., 1981, V.1, 361-380.

[8] Drinfeld V.G. and Sokolov V.V., Equations of Korteweg-de Vries Type and Simple Lie Algebras, Sov. Math. Dokl., 1981, V.23, 457-462; Lie Algebras and Equations of KdV Type, J. Sov. Math., 1985, V.30, 1975-2036.

[9] McIntosh I., Soliton Equations and Connections with Self-Dual Curvature, in Applications of Analytic and Geometric Methods to Nonlinear Differential Equations, Editor P.A. Clarkson, Kluwer Acad. Publ., 1993, 27-39.

[10] Szmigielski J., On Soliton Content of Self Dual Yang-Mills Equations, Phys. Lett. A, 1993, V.183, 293-300.

[11] Guha P., The $\tau$ Functions of the AKS Hierarchy and Twistor Correspondence, Rev. Math. Phys., 1999, V.11, 981-999.

[12] LaChapelle J. and Legare M., Super-Matrix KdV and Super-Generalized NS Equations from Self-Dual Yang-Mills Systems with Supergauge Groups, Lett. Math. Phys., 1998, V.45, 195202. 
[13] De Groot M.F., Hollowood T.J. and Miramontes J.L., Generalized Drinfeld-Sokolov Hierarchies, Commun. Math. Phys., 1992, V.145, 57-84.

[14] Fehér L., KdV Type Systems and $\mathcal{W}$-Algebras in the Drinfeld-Sokolov Approach, Talk at the Marseille 1995 Conference on $\mathcal{W}$-Algebras, hep-th/9510001.

[15] Delduc F. and Fehér L., Regular Conjugacy Classes in the Weyl Group and Integrable Hierarchies, J. Phys. A, 1995, V.28, 5843-5882.

[16] Fehér L. and Marshall I., Extensions of the Matrix Gelfand-Dickey Hierarchy from Generalized Drinfeld-Sokolov Reduction, Commun. Math. Phys., 1997, V.183, 423-461.

[17] Delduc F., Fehér L. and Gallot L., Nonstandard Drinfeld-Sokolov Reduction, J. Phys. A, 1998, V.31, 5545-5563. 